\title{
Analytical Strategy to Prioritize Alzheimer's Disease Candidate Genes in Gene Regulatory Networks Using Public Expression Data
}

\author{
Shweta Bagewadi Kawalia ${ }^{\mathrm{a}, \mathrm{b}, \uparrow}$, Tamara Raschka ${ }^{\mathrm{a}, \mathrm{c}, \boldsymbol{\uparrow}}$, Mufassra Naz $^{\mathrm{a}, \mathrm{b}, 2}$, Ricardo de Matos Simoes ${ }^{\mathrm{d}}$, \\ Philipp Senger ${ }^{\mathrm{a}, 1}$ and Martin Hofmann-Apitius ${ }^{\mathrm{a}, \mathrm{b}, *}$ \\ ${ }^{a}$ Fraunhofer Institute for Algorithms and Scientific Computing (SCAI), Schloss Birlinghoven, \\ Sankt Augustin, Germany \\ ${ }^{\mathrm{b}}$ Rheinische Friedrich-Wilhelms-Universität Bonn, Bonn-Aachen International Center for Information \\ Technology, Bonn, Germany \\ ${ }^{\mathrm{c}}$ University of Applied Sciences Koblenz, RheinAhrCampus, Remagen, Germany \\ ${ }^{\mathrm{d}}$ Dana-Farber Cancer Institute, Boston, MA, USA
}

Accepted 9 June 2017

\begin{abstract}
Alzheimer's disease (AD) progressively destroys cognitive abilities in the aging population with tremendous effects on memory. Despite recent progress in understanding the underlying mechanisms, high drug attrition rates have put a question mark behind our knowledge about its etiology. Re-evaluation of past studies could help us to elucidate molecular-level details of this disease. Several methods to infer such networks exist, but most of them do not elaborate on context specificity and completeness of the generated networks, missing out on lesser-known candidates. In this study, we present a novel strategy that corroborates common mechanistic patterns across large scale $\mathrm{AD}$ gene expression studies and further prioritizes potential biomarker candidates. To infer gene regulatory networks (GRNs), we applied an optimized version of the BC3Net algorithm, named BC3Net10, capable of deriving robust and coherent patterns. In principle, this approach initially leverages the power of literature knowledge to extract $\mathrm{AD}$ specific genes for generating viable networks. Our findings suggest that AD GRNs show significant enrichment for key signaling mechanisms involved in neurotransmission. Among the prioritized genes, wellknown AD genes were prominent in synaptic transmission, implicated in cognitive deficits. Moreover, less intensive studied AD candidates (STX2, HLA-F, HLA-C, RAB11FIP4, ARAP3, AP2A2, ATP2B4, ITPR2, and ATP2A3) are also involved in neurotransmission, providing new insights into the underlying mechanism. To our knowledge, this is the first study to generate knowledge-instructed GRNs that demonstrates an effective way of combining literature-based knowledge and data-driven analysis to identify lesser known candidates embedded in stable and robust functional patterns across disparate datasets.
\end{abstract}

Keywords: Alzheimer's disease, gene regulatory networks, microarray analysis, synaptic transmission

\footnotetext{
I These authors contributed equally to this work.

${ }^{1}$ Current address: Translational R\&D, Bayer CropScience, Monheim, Germany

${ }^{2}$ Current address: Institute of Business \& Information Technology, University of Punjab, Lahore
}

\footnotetext{
*Correspondence to: Prof. Dr. Martin Hofmann-Apitius, Head of the Department of Bioinformatics, Fraunhofer Institute for Algorithms and Scientific Computing (SCAI), 53754 Sankt Augustin, Germany. Tel.: +49 224114 2802; Fax: +49 224114 2656; E-mail: martin.hofmann-apitius@scai.fraunhofer.de.
} 


\section{INTRODUCTION}

Alzheimer's disease (AD) is a very complex idiopathic disease contributing to immense personal and societal burden, with $\sim 13.8$ million people being affected by 2050 [1] in the US alone. High failure rate of AD drugs (98\%) in Phase III trials have resulted in no new FDA approved drugs since 2003 [2]. Moreover, the five previously approved AD drugs just provide symptomatic relief [3]. Not all, but a substantial proportion of these studies focused on amyloid- $\beta$ $(\mathrm{A} \beta)$ and tau accumulations as being synonymous to the AD pathology [4], leading to an unprecedented wealth of molecular and clinical data. Despite the disappointing outcome of the clinical trials, neurology researchers still believe in the definiteness of these two hypotheses [5]. This reaffirms that pathological mechanisms underlying $\mathrm{AD}$ are much more complex than the current consideration, thus, opening up possibilities for new therapeutic targets. Working toward unraveling dysregulated events heralding known and unknown patterns could fill the gaps between $\mathrm{AD}$ hallmarks [6].

Existing experimental data, not being fully exploited, contain compelling evidence that have the potential to contribute next groundbreaking discoveries. The great challenge, however, lies in harmoniously integrating these data and interpreting them differently to derive new-novel insights while maintaining the biological connections. The term "Horizontal Meta-analysis" implies the integration of results from several independent studies [7], thereby increasing the statistical power of the derived conclusion. A more conventional gene-centric approach is to intercross differentially expressed (DE) genes across studies based on majority voting [8], merging gene ranks [9], and combining $p$-values [8]. However, differing factors can lead to a low overlap and discrepancies between studies such as the applied statistical methods, different platforms of the quantitative measurements, and heterogeneity of the patient cohorts [10]. Moreover, these approaches do not shed light on the coordinated genes that collectively orchestrates the underlying (patho-)mechanism. A more consistent and robust approach is through functional enrichment of the dysregulated genes using KEGG [11], MSigDB [12], and other sources of pathway knowledge. However, these approaches have a tendency to converge toward genes that express in large magnitudes and generated hypotheses are restricted by current understanding of pathways.
Network-based approaches that rely on the coherence of expression changes between functionally dependent genes could provide an effective means to overcome the above-mentioned challenges. Such inferred networks have the capability to determine subtle expression shifts between correlated gene pairs that are linked to the dysregulation events. Particularly, these signatures are largely consistent across different studies; thus, emphasizing on its benefits for large scale meta-analysis. In the last few years, we have seen a swarm of methods that infer such networks based on co-expression, regulation, and causal information namely WGCNA [13], BC3Net [14], MRNET [15], ARACNE [16], GENIE3 [17], and CLR [18]. Among these, WGCNA and the bagging version of $\mathrm{C} 3 \mathrm{Net}$ (BC3Net) are popular and computationally efficient methods. BC $3 \mathrm{Net}$ is an ensemble method that statistically infers GRNs based on the strongest mutual dependencies between genes, whereas WGCNA clusters genes on the basis of calculated pairwise correlation coefficients. In the meantime, $\mathrm{BC} 3 \mathrm{Net}$ has been reported in providing meaningful biological insights for large-scale studies [19, 20].

Traditional GRNs identify patterns through differential co-expression analysis [21-23], displaying grouping of patterns based on dysregulated and coordinated biomolecular changes. Integration of such priors drastically improves the context specificity of the inferred networks relative to using data as the sole source [24-26]. However, these spurious discriminative structures, in a given disease context, may vary since DE genes are highly inconsistent across studies [27]. Biologically speaking, one may argue that the differences in functionally enriched components, derived from DE genes, are more consistent than gene-centric activities [28]. But this approach misses out on less informative and less studied non-DE genes, which act in groups, contributing to the observed phenotype or a part of cascade effect. Furthermore, overlaying the inferred networks with known interactions, cataloged in databases or harvested from published literature expand the knowledge space [29, 30]. However, an intriguing question on completeness, veracity and context specificity of these interactions has proven to be a major setback [31].

Here we propose a new approach to identify common signature patterns across public AD studies and prioritize lesser known AD candidates that unravel the general principles of the intrinsic patho-mechanisms. To identify AD mechanistic 

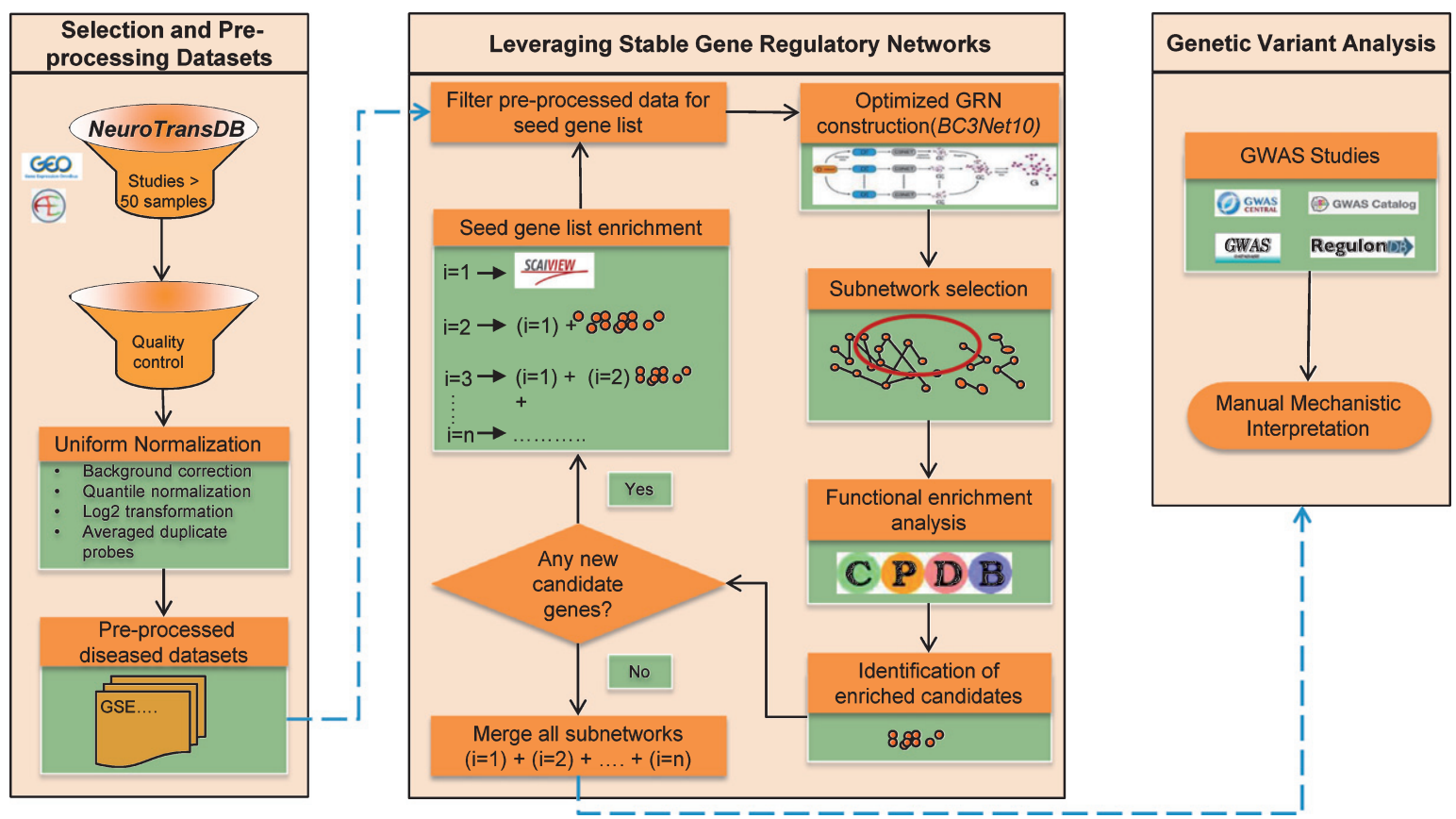

Fig. 1. The overall strategy applied to obtain robust gene expression patterns across public Alzheimer's disease studies. Firstly, four gene expression datasets were shortlisted from NeuroTransDB database. The selected studies underwent preprocessing and quality control. In each dataset, the intensity values were limited to the seed gene list. To enrich the seed, functional enrichment was applied where genes from the identified significant pathways from each dataset's subnetwork (edge weight $>0.5$ ), generated using BC 3 Net10 approach, were included. When no additional genes were identified, subnetworks from each iteration, separately for each dataset, were merged into an aggregated network for further prioritization of the genes using genetic variant analysis.

footprints, we established an optimized workflow around $\mathrm{BC} 3 \mathrm{Net}$ to extract more robust and coherent co-expressed gene patterns (named BC3Net10). The approach allows us to converge lesser known candidates into the final generated GRNs. Moreover, to generate context-specific GRNs, the main rationale applied was to leverage the power of prior knowledge and functionally enriched candidates in the data. First, we identified the most frequently discussed genes in the scientific literature using our literature mining environment SCAIView; this is called the "seed". We are aware that the generated GRNs may be biased due to the incomplete nature of the prior knowledge. To overcome this limitation, we extended the seed by adding all the genes from the enriched pathways, determined for the high scoring inferred interactions from BC3Net10. Several iterations are performed until there are no more genes to be added to the seed. Finally, an aggregated GRN from all the iterations, for each dataset, is generated to prioritize functional context and determine lesser known candidates from the genetic variant analysis. Figure 1 presents an overview of the strategy in this study, and descriptions of the methodology are available in the
Material and Methods section. This work suggests a context-specific strategy for future interpretation of the GRNs. Taken together, our work demonstrates that optimizing the GRN generation can provide a powerful resource to prioritize novel candidate genes (could serve as biomarkers) and common functional components that axles the disease progression.

\section{MATERIAL AND METHODS}

\section{Selection of datasets}

We collated eight AD datasets (cf. Table 1) that are composed of 50 or more samples (for diseased and control phenotype) from the previously developed value-added database, NeuroTransDB [32]. Briefly, this database contains manually curated metadata annotations for eligible neurodegenerative studies. The datasets have been harvested from publicly available resources namely, Gene Expression Omnibus (GEO) [33] and ArrayExpress [34], using a keywordbased search approach.

Furthermore, datasets that fulfilled the following criteria were retained for generating gene regulatory 
Table 1

List of datasets shortlisted from NeuroTransDB database for generating gene regulatory networks. Final selected studies are highlighted in bold

\begin{tabular}{|c|c|c|c|c|c|}
\hline \multirow[t]{2}{*}{ GEO ID } & \multicolumn{2}{|c|}{ Number of Samples } & \multirow[t]{2}{*}{ Sample Source } & \multirow[t]{2}{*}{ Stage } & \multirow[t]{2}{*}{ Platform } \\
\hline & Diseased & Control & & & \\
\hline GSE5281 & 87 & 74 & $\begin{array}{l}\text { Entorhinal cortex, Hippocampus, } \\
\text { Primary visual cortex, Prefrontal } \\
\text { cortex, Medial temporal gyrus, } \\
\text { Superior frontal gyrus }\end{array}$ & - & Affymetrix HG U133 Plus 2 \\
\hline GSE44768 & 129 & 101 & Cerebellum & LOAD & Rosetta/Merck Human 44k 1.1 microarray \\
\hline GSE44771 & 129 & 101 & Visual cortex & LOAD & Rosetta/Merck Human 44k 1.1 microarray \\
\hline GSE44770 & 129 & 101 & Dorsolateral prefrontal cortex & LOAD & Rosetta/Merck Human 44k 1.1 microarray \\
\hline GSE13214 & 52 & 40 & Hippocampal, Cortex frontal & Braak 4-6 & Homo sapiens 4.8K 02-01 amplified cDNA \\
\hline GSE15222 & 176 & 187 & Cortical tissue & LOAD & Sentrix HumanRef-8 Expression BeadChip \\
\hline GSE29676 & 350 & 200 & Blood & - & Invitrogen ProtoArray v5.0 \\
\hline GSE33528 & 615 & 600 & Blood & LOAD & $\begin{array}{l}\text { Illumina Human- Hap650Yv2 Genotyping } \\
\text { BeadChip }\end{array}$ \\
\hline
\end{tabular}

networks: (1) oligonucleotide arrays for analysis consistency, (2) availability of raw data to facilitate uniform pre-processing, and (3) expression profiling carried out on brain tissue. A list of four potential datasets that comply with the above conditions is eligible for further analysis: GSE5281, GSE44771, GSE44770, and, GSE44768. An overview of the platform, stage, and brain region information for the same is given in Table 1. Among these, GSE44771, GSE44770, and GSE44768 were from a single study reported by Zhang et al. [35] for late-onset AD.

\section{Pre-processing and gene annotation}

The four selected datasets were processed identically to reduce variance and to maintain consistent quality. All analysis was carried out with R (Version 3.1.3) [36], an open-source statistical language, using the packages from Bioconductor (Version 3.0) [37]. The overall step-by-step workflow is shown in Fig. 1. To eliminate the variance effect of non-specific hybridization, all the downloaded raw data were uniformly normalized by performing background correction, quantile normalization, and averaging the expression values of duplicate probes on $\log 2$-transformed intensity values. For Affymetrix platform, robust multi-array average method ( $r m a)$ [38] available in Bioconductor package affy was applied. Similar methods available in Bioconductor package limma [39] were applied on Rosetta/Merck Human 44k 1.1 microarray chip.

Affymetrix probes to gene symbols annotation mapping were obtained from the "hgu133plus2.db" Bioconductor package. In the case of Rosetta/Merck chip, the gene symbol annotations were provided directly along with the intensity values. For multiple probes mapping to the same gene within an array, average expression values were used. Unmapped probes were excluded from further analyses. As a result of this preprocessing step, we retained 20155 in GSE5281, 11254 in GSE44771, 10437 in GSE44770, and 12000 in GSE44768 genes for further analysis.

\section{Quality control and outlier detection}

Using the Bioconductor package arrayQualityMetrics [40], we assessed the array quality and removed the outlier samples. Describing shortly, arrayQualityMetrics determine outliers using three different metrics: (1) distance between samples using principal component analysis, (2) array intensity distributions of all samples on the array; and (3) individual array quality through MA-plots. If a sample is detected as an outlier in either of the three metrics, we discard it from further analysis. In the four selected datasets, 9 in GSE5281, 19 in GSE44771, 27 in GSE44770, and 12 in GSE44768 arrays were outliers. The list of identified outlier arrays is provided in the Supplementary File 3. The remaining arrays that passed the quality control were processed as described earlier.

\section{Leveraging stable gene regulatory networks}

In order to derive $\mathrm{AD}$ relevant GRNs, we divided the AD gene expression profile based on their phenotypes, disease and normal. Subsequently, BC3Net10 algorithm was applied only on diseased samples for AD seed genes, cf. Fig. 1. GRNs were generated independently for each dataset, visualized as igraph objects in Cytoscape tool [41]. Network topological properties such as node degree, hub genes, etc. were determined using the Bioconductor package igraph. 


\section{Filter pre-processed data for seed gene list}

Prior to GRN generation, each pre-processed dataset was restricted to the genes in the seed. Initially, it consists of a set of literature-derived genes that have high probability of direct or indirect involvement in $\mathrm{AD}$ pathogenesis (see the section on Gathering initial seed genes). The rationale behind applying this filtration is to maintain the disease specificity and reduce high run time due to bootstrapping in BC3NET. Further on, after every functional enrichment iteration, we again restrict the expression data to the new seed.

\section{Gathering initial seed genes}

The backbone of the seed comprises of the results harnessed from our text-mining knowledge framework, SCAIView [42]. SCAIView is a knowledge discovery framework that supports named entity recognition, information retrieval, and information extraction on large textual sources. Its capability to rank documents and biomedical entities based on the relevancy score allows retrieval of significant players in a disease context [43, 44]. Querying SCAIView for $\mathrm{AD}$ related genes resulted in 4808 genes, as of 2 January 2016. Only the top 500 retrieved genes were used as the initial seed, depicted as $i=1$ in Fig. 1.

\section{Optimized GRN construction}

For the construction of GRNs, R package bc3net was applied to the processed data with 100 bootstraps $(\mathrm{B}=100)$. Briefly explained, one aggregated network was generated by applying the C3Net algorithm on 100 bootstrapped data, which were inferred from given processed dataset. Statistically, non-significant edges inferred by $\mathrm{C} 3 \mathrm{Net}$ and $\mathrm{BC} 3 \mathrm{Net}$ were discarded using Bonferroni's multiple testing correction, $\alpha=0.05$. In the resulting aggregated network, edge weights represent the frequency of a correlated gene pair in 100 random sampling, ranging from 0 to 1 .

During random sampling, true and most prominent correlations are stochastically more likely to be selected than the non-correlated ones. This is reflected in BC3Net networks, where three independently generated GRNs, inferred from the same gene expression dataset (GSE5281), have an edge overlap of $\sim 74 \%$ (for no edge weight cutoff) and $\sim 89 \%$ (for edge weight $\geq 0.5$ ); the node overlap always remained $100 \%$. The $\mathrm{BC} 3 \mathrm{Net}$ parameters used for performing this analysis are: boot $=100$, estimator = "pearson", disc = "equalwidth", $\mathrm{mtc} 1=$ TRUE, alpha1 $=0.05, \operatorname{adj} 1=$ "bonferroni”, $\mathrm{mtc} 2=$ TRUE, alpha $2=0.05, \operatorname{adj} 2=$ "bonferroni", weighted
$=$ TRUE, igraph $=$ TRUE, verbose $=$ FALSE and number of seed genes $=4808$ (see the section on Gathering initial seed genes). However, less frequently appearing, yet plausible, edge interactions could offer the potential for promising candidates that are buried in expression data.

We observed that the intersection between independently generated GRNs saturated after 5-10 repetitions of the $\mathrm{BC} 3 \mathrm{Net}$ algorithm on the same dataset. Thus, in order to expand the knowledge space around $\mathrm{AD}$ candidates and for completeness, we propose an optimization of the randomness to devise a more recall optimized GRNs. More specifically, we applied the BC3Net algorithm to the same dataset 10 times, named BC3Net10. Finally, we aggregated the 10 independently generated GRNs into one. The final edge weight is now the mean of the computed edge score from 10 GRNs. This increases the prospect of deducing more reasonable functional speculations in complex diseases with the high probability of novelty for further investigations.

\section{Subnetwork selection and functional enrichment analysis}

The choice of a threshold can significantly affect the integrity of the network and the co-expression modules derived from it. In this regard, computed edge weight (mean weight $>0.5$ ) from $\mathrm{BC} 3 \mathrm{Net} 10$ was used as the filter criteria for selecting significant gene pairs in the generated GRNs. This increases the significance level by $50 \%$ for the inferred interactions in each dataset.

The overlap between the inferred interactions/edges was very low (zero genes common to all 4 subnetworks, see Fig. 2) when BC3Net was applied on the initial seed. Several reasons can be presumed for lack of common and stable genes such as different platforms, distinct brain tissues, diverse patient cohort, and treatment heterogeneity. However, numerous studies have already shown that the functional signatures are more stable relative to individual gene level information [45-48]. In this context, to extract the most representative biological pathways for genes in the subnetworks (separately for each dataset), we performed functional enrichment analysis (based on one-sided Fisher's exact test) for KEGG pathway information using ConsensusPathDB (CPDB) [49] (Release 30). Using the Bioconductor package, org.Hs.eg.db [50], we mapped the gene symbols to Entrez gene identifiers obtained from CPDB. 


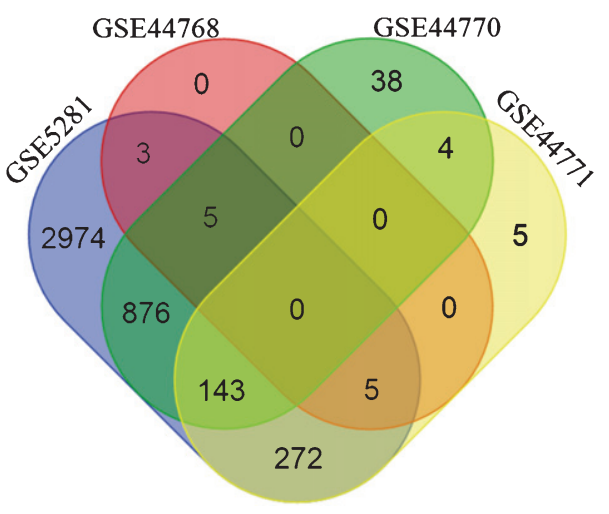

Fig. 2. Venn diagram depicting the gene overlap between the subnetworks (edge weight $>0.5$ ) of the four datasets, generated using the initial seed. The initial seed was compiled from top 500 genes retrieved by querying SCAIView for Alzheimer's disease related genes. It is evident that there are no common genes among the four dataset's subnetworks. Differing factors between platforms, analytical methods, tissue source, etc. could contribute to such a behavior.

\section{Identification of enriched candidates and seed gene list enrichment}

We devised a strategy to expand the seed through functional enrichment analysis of the individual network modules inferred by the GRNs, enabling us to quantify the saturation of the inferred network. We extracted significant pathways (for the $p$-value $<0.05$ ) common between the determined subnetworks of the four datasets, generated using the initial seed. We added a new gene (called enriched candidate) to the seed when the gene belongs to the respective CPDB and KEGG pathway gene set that is significantly enriched across all 4 inferred GRNs and is not present in our initial seed. Further, we repeated the functional enrichment analysis to determine overlapping pathways for the enriched seed. We leveraged the identified enriched candidates in these pathways by subsequent inclusion in the seed iteratively until saturation. Once the seed has reached its saturation, we merge the networks of all iterations, separately for each dataset, to generate an aggregated network. This approach goes beyond just candidate enrichment, corresponding to a maximal $\mathrm{AD}$ specificity with minimal noise and harvesting lesser known genes in GRNs.

\section{Gene list prioritization by genetic variant analysis}

For the consensus network, we identified genes (involved in significant pathways and hub genes) to prioritize them using genetic variant analysis. Multiple genetic variants are attributed in the etiology of complex diseases. To investigate the impact of genetic variation, we extracted $\mathrm{AD}$ evidences for single-nucleotide polymorphisms (SNPs) from GWAS catalog [51], GWAS Central [52], and gwasDB [53], resulting in 11,314 SNPs. Further, linkage disequilibrium (LD) analysis was carried out to enrich the list of $\mathrm{AD}$ associated genetic variants, which were sorted based on their chromosome location. LD is SNP's property on a contiguous stretch of a chromosome that describes the degree to which an allele of one genetic variant is inherited or correlated with an allele of another genetic variant within a population. The LD analysis was performed using HaploReg v2 (developed by Broad Institute of MIT) [54] based on dbSNP-137 [55], motif instances (based on PWMs provided by the ENCODE project database) [56], enhancer annotations (adding 90 cell types from the Roadmap Epigenome Mapping Consortium) [57], and eQTLs (from the GTex eQTL browser) [58]. With LD threshold cutoff of $\mathrm{r} 2=0.8$, we obtained 115,782 SNPs. Further on, these SNPs were filtered based on theENSEMBL SNPEffect predictor that estimates the influence of SNP variants on the respective transcripts of a gene and their gene products [59], shortlisting 4,831 SNPs. Genes obtained from the aggregated networks were boiled down to those associated with shortlisted SNPs. Finally, these refined genes were ranked using a cumulative score of their SNPs from RegulomeDB [60], dbSNP's functional annotation [55], ENSEMBL's Variant Effect Predictor [61], and regulatory feature annotation by ENSEMBL variant database [62]. RegulomeDB's ranking is based on the functional annotations from ENCODE database [63], chromatin states from the Roadmap Epigenome Consortium [57], DNase-footprinting [64], position weighted matrix for transcription factor binding [65], and DNA methylation [66].

\section{RESULTS AND DISCUSSION}

\section{Algorithm convergence and network properties}

Under the premise that lesser known genes are not prominently represented in literature, we extended the set of seed genes through functional enrichment (see the section on Subnetwork selection and functional enrichment analysis). As depicted in Fig. 1, BC3Net10 was applied on the identified four datasets 
Table 2

Statistics of the iterative functional enrichment approach

\begin{tabular}{llll}
\hline Iteration (i) & Seed & $\begin{array}{l}\text { No. of overlapping } \\
\text { pathways between the } \\
\text { four datasets }\end{array}$ & $\begin{array}{l}\text { No. of enriched candidate } \\
\text { genes obtained from the } \\
\text { overlapping pathways }\end{array}$ \\
\hline 1 & 10 & 820 \\
2 & SCAIView (500) & 38 & 1148 \\
3 & i $1+820$ & 30 & 361 \\
4 & i $2+1148$ & 30 & 84 \\
5 & i $3+361$ & 32 & 41 \\
6 & i $4+84$ & 33 & 7 \\
7 & i5 +41 & 37 & - \\
\hline
\end{tabular}

for different seed lists to generate AD GRNs. Iteration 1, where we generated GRNs for SCAIView genes, resulted in 10 overlapping and significant pathways between the four datasets. From these pathways, we obtained 820 genes that were earlier not present in the seed. Hence, there is a clear need for further enrichment of the seed, which is done by including these newly identified candidates to the seed and repeating the functional enrichment step. In the second iteration, we identified 38 overlapping significant pathways between the datasets. This iteration continues seven times until there are no newer candidates to be added. Table 2 provides the statistics of the number of pathways identified in each iteration, along with the number of enriched candidate genes that were added to the seed. A detailed list of the enriched candidates (as HGNC symbols) identified in each iteration is provided in Supplementary File 1. A sharp increase in the number of enriched candidates is observed in the first two iterations, which drops to zero in the seventh iteration. We assume that this indicates the completeness of the gene set that belongs to $\mathrm{AD}$, specific to the selected four datasets.

Extension of the GRNs using enriched seed is a knowledge guided approach, which relies on the functional information derived from the gene expression data. We note that the GRNs grow progressively, both inferred interactions and the participating candidates, but in the process, eliminates few of the previously inferred interactions. A potential reason is that the extension of the expression matrix with new seed contributes to a shift in the significance of the inferred interactions by BC3Net. It implies that although we obtain a final GRN for saturated seed (iteration 7), aggregating networks from earlier iterations could capture interactions that were previously inferred as potential. The fraction of nodes and edges from each iteration that makes up the aggregated network, for each dataset, is presented in Fig. 3. We observe that the addition of nodes, in each iteration, across datasets, remained stable whereas the same cannot be said for the edges. The variance in edges could be presumed that the newly added set of genes bring in higher functional relevance through newly inferred interactions in one or the other iteration.

An assessment of the completeness of a GRN for $\mathrm{AD}$ specific genes can be precisely estimated by plotting the mean and the variance of the number of nodes and edges present in each dataset for each iteration. From Fig. 4a, it is evident that the enrichment of the most relevant genes reach saturation. This increases the statistical significance of the GRNs suggesting an increment in the biological confidence. It is apparent that not all the genes present in the seed agree across platforms due to various differing experimental factors. However, we expect functional signatures across the datasets to be more agreeable. Analyzing edges (see Fig. 4b), we observe that they orient three times, at saturation, to the number of nodes. The relative higher number of edges demonstrate that the gene sets are highly related, showing immense inter-connectivity between several functional modules. The high variance observed, in both nodes and edges, is contributed by the large network size of GSE5281 relative to the other three datasets. Details of the number of nodes and edges present in each dataset at each iteration is provided are Supplementary File 2.

\section{Hub genes}

Hub genes have a higher grade of lethality when dysregulated in a pathological condition, referred to as centrality lethality rule [67]. For each aggregated GRN, a gene was defined as a hub gene when it had a higher degree of distribution ( $>95 \%$ quantile). By this criterion, we identified 29 in GSE5281, 8 in GSE44768, 14 in GSE44770, and 1 in GSE44771 as hub genes. Table 3 displays the list of identified hub 


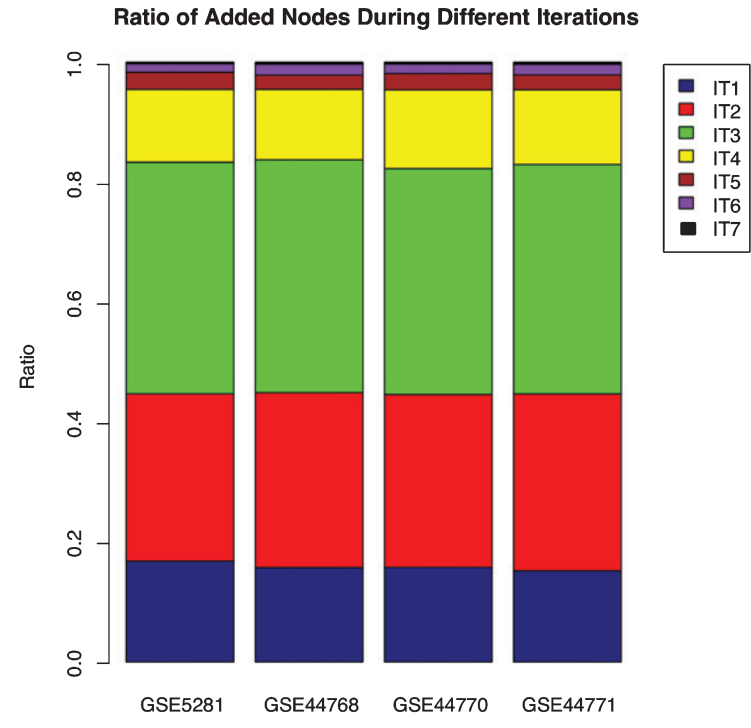

(a)

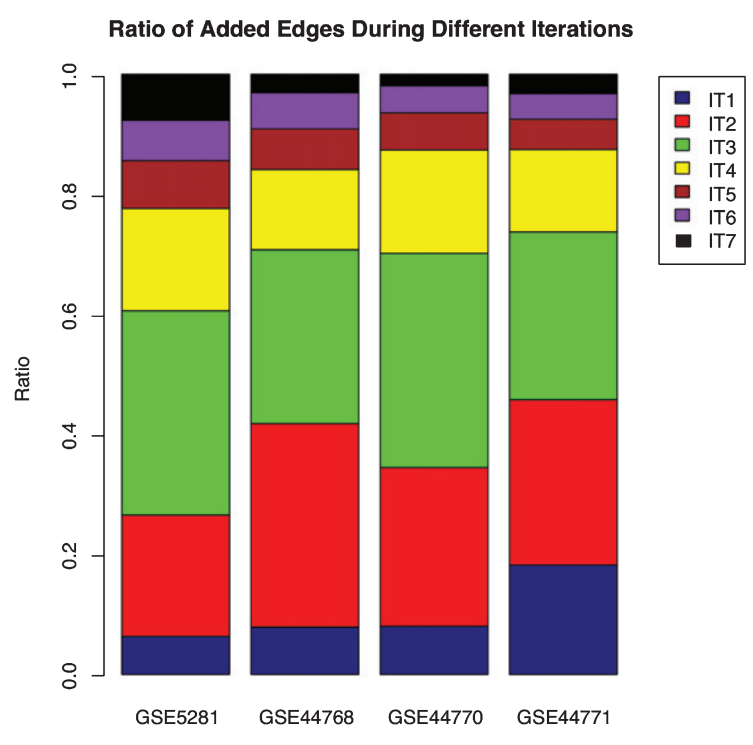

(b)

Fig. 3. Stratification of the nodes and edges in four aggregated networks. Each stack in the bar plot represents the fraction of nodes added in that iteration (IT) relative to the aggregated network (considered as 1). The addition of nodes remained stable across the datasets in each iteration. However, the inclusion of edges varies, which could be presumed due to newly inferred interactions from the newly included nodes in each iteration. (a) Fraction of added nodes in different iterations; (b) Fraction of added edges in different iterations.

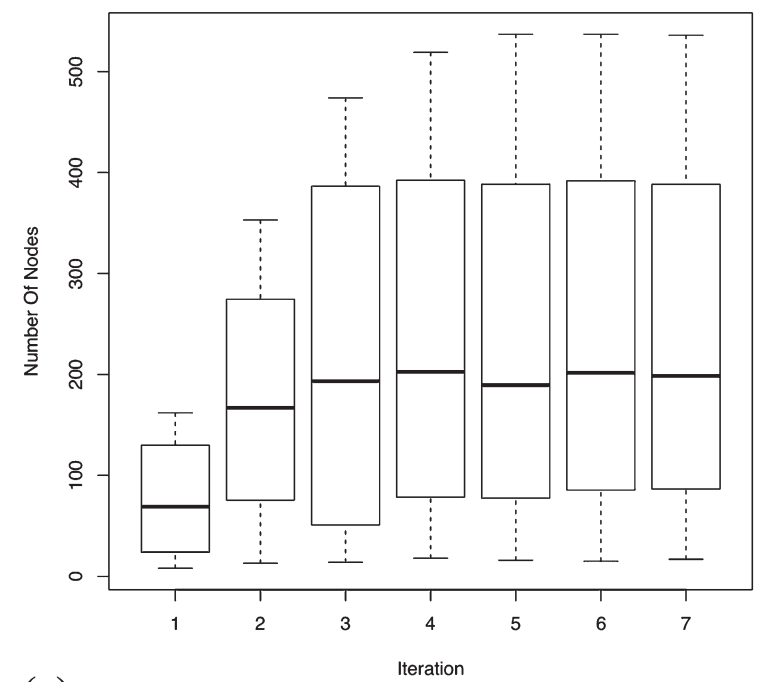

(a)

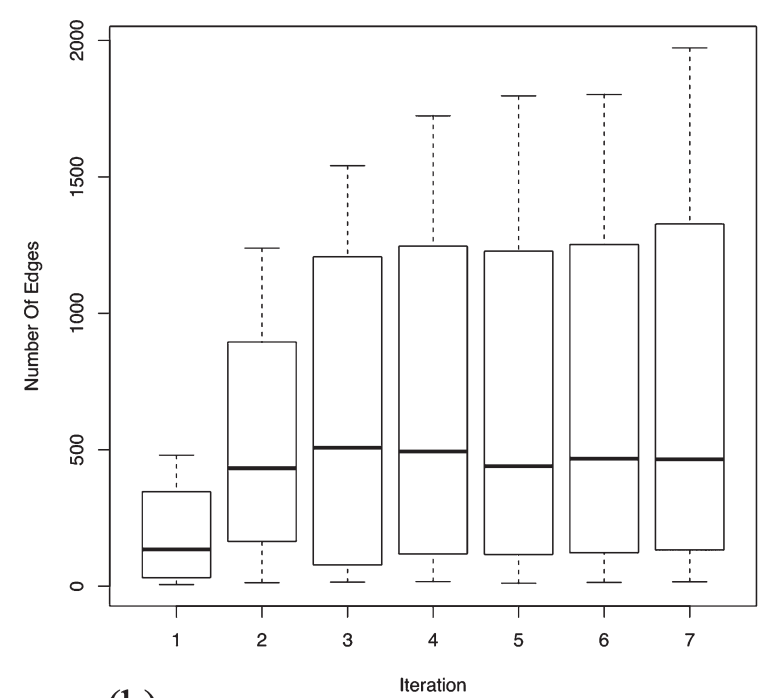

(b)

Fig. 4. Mean and variance distribution across four datasets for the added nodes and edges in each iteration. Enrichment of nodes and edges reach saturation after 7 th iteration, suggesting the completeness of the generated GRNs. Relatively high number of edges (see y-axis range) show immense inter-connectivity between the genes in the GRNs. (a) Boxplot for mean and variance distribution of nodes; (b) Boxplot for mean and variance distribution of edges.

genes along with their node degree and pathway annotation (only for significant pathways, see Functional homogeneity across datasets section). Interestingly, there were no common hub genes between the four datasets. It was evident that six of the hub genes were perturbed in multiple pathways. Many of the hub genes were functionally enriched in neurotrophin signaling, endocytosis, and estrogen signaling path- 
Table 3

Hub genes identified in the aggregated network for the four datasets. The genes are sorted by their hub degree within each dataset. Only significant pathways are listed here (see Table 4 for the list)

\begin{tabular}{|c|c|c|c|c|}
\hline GEO ID & Gene Symbols & Hub Degree & Pathway Annotation (CPDB) & $\begin{array}{l}\text { Similar results in } \\
\text { other datasets? }\end{array}$ \\
\hline \multirow[t]{23}{*}{ GSE5281 } & HFE & 244 & - & - \\
\hline & ATP2A3 & 162 & $\begin{array}{l}\text { Calcium signaling, pancreatic } \\
\text { secretion }\end{array}$ & - \\
\hline & GLP1R & 150 & Insulin Secretion & - \\
\hline & ADRBK1 & 145 & Endocytosis & GSE44770 \\
\hline & CACNG4, CACNG6 & 141 & - & - \\
\hline & KCNJ5 & 132 & Estrogen signaling & - \\
\hline & $\mathrm{P} 2 \mathrm{RX} 2$ & 130 & Calcium signaling & GSE44770 \\
\hline & KPNA2 & 122 & - & - \\
\hline & NOX1 & 118 & - & - \\
\hline & CACNG5 & 113 & - & - \\
\hline & EPN1 & 113 & Endocytosis & - \\
\hline & WAS & 112 & - & - \\
\hline & CASP10 & 111 & Apoptosis & - \\
\hline & HSPB6, EPHA4 & 109 & - & - \\
\hline & ADNP & 108 & - & - \\
\hline & DNAH3 & 106 & - & - \\
\hline & GRIN2A & 105 & Calcium signaling & - \\
\hline & UBQLN1 & 101 & - & - \\
\hline & IL34, ATP5A1, UBE2L3 & 100 & - & - \\
\hline & DPYSL2 & 99 & - & - \\
\hline & FOLR2 & 98 & Endocytosis & - \\
\hline & NPR1 & 96 & - & - \\
\hline & DNM1L, KLC1, ATP5G3 & 92 & - & - \\
\hline \multirow[t]{8}{*}{ GSE44768 } & RASGRF1 & 80 & - & - \\
\hline & DNAL4 & 63 & - & - \\
\hline & EPHA1 & 60 & - & - \\
\hline & CHRND & 59 & - & - \\
\hline & TRPC1 & 54 & Pancreatic secretion & GSE5281, GSE44770 \\
\hline & PAK7 & 50 & - & - \\
\hline & NDUFA4 & 44 & - & - \\
\hline & CHMP4B & 44 & Endocytosis & - \\
\hline \multirow[t]{13}{*}{ GSE44770 } & IVNS1ABP & 103 & - & - \\
\hline & FGF18 & 92 & - & - \\
\hline & ATF2 & 90 & Estrogen signaling, Insulin secretion & - \\
\hline & CTSG & 88 & - & - \\
\hline & GABRE & 86 & - & - \\
\hline & FBXL2 & 81 & - & - \\
\hline & GAPDH & 75 & - & - \\
\hline & DIO1 & 72 & Thyroid hormone signaling & - \\
\hline & $\mathrm{CACNB} 3, \mathrm{CDK} 2$ & 66 & - & - \\
\hline & NFKBIB & 66 & $\begin{array}{l}\text { Adipocytokine signaling, } \\
\text { neurotrophin signaling, NOD-like } \\
\text { receptor signaling }\end{array}$ & GSE44768 \\
\hline & PRDM4 & 64 & Neurotrophin signaling & - \\
\hline & MAPK9 & 63 & $\begin{array}{l}\text { Adipocytokine signaling, } \\
\text { neurotrophin signaling, NOD-like } \\
\text { receptor signaling }\end{array}$ & - \\
\hline & PIK3CB & 63 & $\begin{array}{l}\text { Apoptosis, estrogen signaling, } \\
\text { neurotrophin signaling, thyroid } \\
\text { hormone signaling }\end{array}$ & GSE5281 \\
\hline GSE44771 & HSPA2 & 18 & Endocytosis, estrogen signaling & - \\
\hline
\end{tabular}

ways. Additional associated pathways with hub genes include calcium signaling, adipocytokine signaling, NOD-like receptor signaling, insulin signaling, apoptosis, thyroid signaling, and pancreatic secretion. The majority of these hub genes formed a connected subnetwork within each dataset, indicative of a possible cooperative effect in AD pathology (see Supplementary Figure 1). In the case of GSE44771, due to the 
presence of a single hub gene, we extracted the largest subnetwork associated with HSPA2.

\section{Functional homogeneity across datasets}

Are the core functional modules (set of interconnected-genes) unique to a human brain region or do they depict patterns reflecting the tight linkage between different regions of the brain? To address these questions, we compared the final determined significant pathways across the four aggregated GRNs (outlined in Methods). The functional enrichment analysis revealed 187 in GSE5281, 120 in GSE44768, 170 in GSE44770, and 43 in GSE44771 inferred modules within significant KEGG pathways. We computed a simple overlap between the four GRNs to assess the conserved pathways, resulting in 34 pathways. Because this list contained pathways that were not directly relevant to the core pathophysiology of $\mathrm{AD}$, we categorized them into subsets based on their pertinence to $\mathrm{AD}$, see Table 4. Please refer to Supplementary File 4 for details of summary statistics. From these, we chose to focus on pathways that exacerbate the AD phenotype, classified as "Potential". Table 4 also provides the statistics of the number of genes enriched for these pathways in each dataset. Interestingly, there are no common genes between the four datasets when compared at the pathway level. However, many of the genes are shown to be involved in more than one potential pathway, providing the basis for functional connectivity in $\mathrm{AD}$.

\section{Regulatory underpinning across Consensus network}

As described in the section on Functional homogeneity across datasets, the genes in different GRNs are complementary for the top significant pathways. Thus, to provide a broader coverage than a single GRN and to infer stronger relationships through consensus, we merged the four aggregated GRNs into one, called consensus network. What we expect is to uplift the most promising pathways due to the assembly of more participating genes. To assess the concept of functional enrichment, we plot the $p$-values of all the significant pathways, listed in Table 4, for each of the aggregated and consensus GRNs, see Fig. 5. From the figure, it is evident that these pathways have attained higher significance level (better $p$-values) in consensus GRN due to the gene complementarity from the aggregated GRNs.

\section{Prioritizing through genetic variant analysis}

We compiled 608 genes from listed significant pathways across datasets (see Table 4) and hub genes. We mapped these genes to the 4,831 shortlisted ENSEMBL SNPs (see Methods). For the obtained 167 mapped genes, we ranked them based on the calculated cumulative score for their potential functional consequences in a disease context. Restricting the ranked genes to the RegulomeDB score of 3, we generated a final list of 44 high ranked genes. In addition, we looked into the AD GWAS meta-analysis study carried out by Lambert et al. [68]. Among all their listed genes carrying genetic AD risks, we found three (AP2A2, DPYSL2, and EPHA1) of them to be present in our 608 gene list, including one (EPHA1) newly reported in their study; these three were added to our final gene list. Please refer to Table 5 for detailed ranking and RegulomeDB score. Additional investigation revealed 14 out of 47 genes from our final gene list are either validated by eQTLs studies or experimentally evident that the SNPs are linked to the active promoter region of the gene. These genes include IL1B, NSF, HLA-F, NOTCH4, VCL, PSAP, STX2, GGA2, STK11, CSF3R, LMNA, CTNNA2, HLA-C, and RAB11FIP4. When we performed a comprehensive analysis of the biomedical literature, we found that many of these genes had no evidence of being linked to $\mathrm{AD}$, but were rather known to be involved in AD co-morbidity diseases (see Supplementary Table 1).

\section{Well known prioritized $A D$ candidates}

Apart from the new novel candidates, our method also determined well-known candidates (nearly 50 articles in AD) such as IL1B, NTRK2, GRIN2A, FYN, and DPYSL2. The IL1B gene is a pro-inflammatory cytokine that has been long studied for its modulatory effect in AD. It is reported that the expression of IL1B significantly increases with the increase of AD-related neurofibrillary pathology [69]. Synaptic plasticity, such as long-term potentiation, is crucial for learning and memory. A neurotransmitter modulator, BDNF, mediates neuronal survival and plasticity by regulating neurotrophins through NTRK2. AD patients with cognitive deficits have been accounted with reduced levels of BDNF [70-72]. Similarly, GRIN2A is a subunit of NMDA receptors, whose reduced expression increases the vulnerability of neurons to excitotoxicity in $\mathrm{AD}$, correlated with cognitive impairment due to reduced plasticity $[73,74]$. A strong corre- 
Table 4

Landscape of significant pathways $(p$-value $<0.05)$ determined across datasets

\begin{tabular}{|c|c|c|c|c|c|c|c|}
\hline $\begin{array}{l}\text { Common } \\
\text { Pathways }\end{array}$ & Pathway Category & $\begin{array}{l}\text { Total no. of genes } \\
\text { in the pathway }\end{array}$ & $\begin{array}{l}\text { Number o } \\
\text { GSE5281 }\end{array}$ & $\begin{array}{l}\text { of genes enric } \\
\text { GSE44768 }\end{array}$ & $\begin{array}{l}\text { ched for the } \\
\text { GSE44770 }\end{array}$ & pathway & Consensus \\
\hline Cancer & Basal cell carcinoma & 55 & 5 & 2 & 7 & 1 & 15 \\
\hline Cancer & Colorectal cancer & 62 & 6 & 2 & 8 & 1 & 14 \\
\hline Cancer & Pathways in cancer & 398 & 64 & 27 & 40 & 3 & 119 \\
\hline Cancer & Small cell lung cancer & 86 & 15 & 4 & 9 & 2 & 27 \\
\hline Comorbidity & Amyotrophic lateral sclerosis & 51 & 14 & 2 & 5 & 1 & 18 \\
\hline Comorbidity & $\begin{array}{l}\text { Arrhythmogenic right ventricular } \\
\text { cardiomyopathy }\end{array}$ & 74 & 13 & 5 & 8 & 2 & 21 \\
\hline Comorbidity & Dilated cardiomyopathy & 90 & 17 & 6 & 7 & 2 & 26 \\
\hline Comorbidity & Hypertrophic cardiomyopathy & 83 & 14 & 7 & 7 & 2 & 23 \\
\hline Comorbidity & Rheumatoid arthritis & 91 & 10 & 4 & 7 & 1 & 20 \\
\hline Infection & $\begin{array}{l}\text { Epithelial cell signaling in } \\
\text { Helicobacter pylori infection }\end{array}$ & 68 & 9 & 2 & 6 & 1 & 16 \\
\hline Infection & Influenza A & 177 & 25 & 4 & 22 & 2 & 46 \\
\hline Infection & Shigellosis & 61 & 12 & 3 & 7 & 1 & 19 \\
\hline Infection & Toxoplasmosis & 120 & 14 & 3 & 12 & 2 & 26 \\
\hline Infection & Tuberculosis & 179 & 21 & 4 & 23 & 3 & 46 \\
\hline Infection & Vibrio cholera infection & 54 & 9 & 2 & 2 & 1 & 13 \\
\hline Infection & Viral myocarditis & 60 & 12 & 2 & 5 & 2 & 19 \\
\hline Others & Melanogenesis & 101 & 18 & 3 & 10 & 1 & 30 \\
\hline Others & $\begin{array}{l}\text { Neuroactive ligand-receptor } \\
\text { interaction }\end{array}$ & 275 & 57 & 24 & 30 & 3 & 98 \\
\hline Potential & Apoptosis & 86 & 14 & 2 & 6 & 1 & 20 \\
\hline Potential & Calcium signaling pathway & 180 & 43 & 12 & 16 & 2 & 62 \\
\hline Potential & Endocytosis & 213 & 47 & 10 & 21 & 4 & 70 \\
\hline Potential & Neurotrophin signaling pathway & 120 & 24 & 6 & 17 & 1 & 44 \\
\hline Potential & NOD-like receptor signaling pathway & 57 & 9 & 3 & 6 & 1 & 16 \\
\hline Potential & PPAR signaling pathway & 69 & 11 & 4 & 9 & 2 & 22 \\
\hline Potential & Synaptic vesicle cycle & 63 & 15 & 4 & 8 & 1 & 26 \\
\hline Potential & Adipocytokine signaling pathway & 70 & 17 & 6 & 8 & 1 & 27 \\
\hline Potential & Insulin secretion & 86 & 18 & 3 & 10 & 1 & 28 \\
\hline Potential & Pancreatic secretion & 96 & 21 & 5 & 9 & 1 & 30 \\
\hline Potential (hormones) & Estrogen signaling pathway & 100 & 23 & 4 & 7 & 1 & 32 \\
\hline Potential (hormones) & Thyroid hormone signaling pathway & 119 & 26 & 3 & 10 & 1 & 37 \\
\hline Potential (others) & Lysosome & 122 & 13 & 7 & 11 & 4 & 33 \\
\hline Potential (others) & Phagosome & 155 & 31 & 4 & 16 & 2 & 48 \\
\hline
\end{tabular}

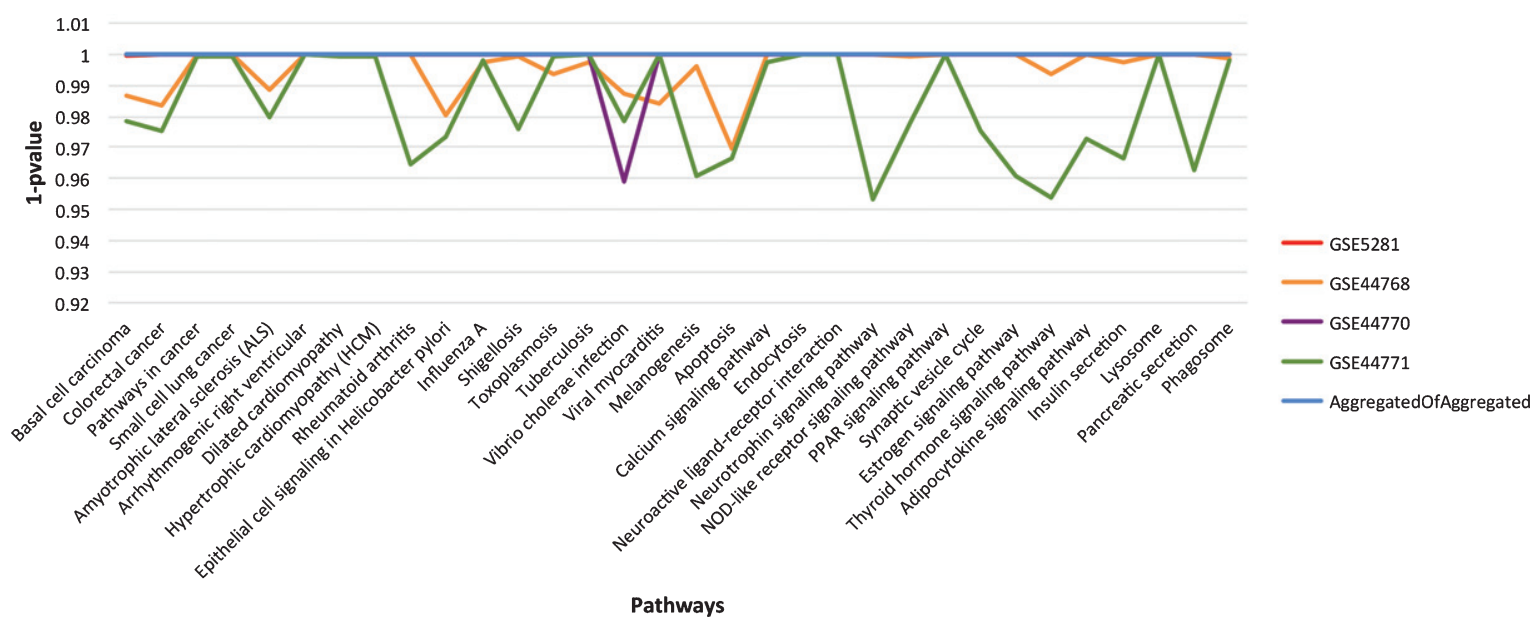

Fig. 5. The landscape of $p$-value for the final list of significant pathways. For easy visualization, we have used 1- $p$ value instead of $p$-value on Y-axis. Each line in the graph represents aggregated GRN for specified dataset (see chart legend). The listed pathways show higher significance level in consensus GRN in comparison to the individual dataset aggregated GRNs. 
Table 5

List of genes prioritized using genetic variant analysis

\begin{tabular}{|c|c|c|c|c|}
\hline Rank & $\begin{array}{l}\text { Gene } \\
\text { Symbol }\end{array}$ & $\begin{array}{l}\text { RegulomeDB } \\
\text { score }\end{array}$ & $\begin{array}{l}\text { No. evidences } \\
\text { for } \mathrm{AD}\end{array}$ & Pathways involved \\
\hline 1 & IL1B & $1 b$ & 1073 & Apoptosis, NOD-like receptor signaling \\
\hline 2 & NSF & $1 d$ & 8 & Synaptic vesicle cycle \\
\hline 3 & HLA-F & 1f & 0 & Endocytosis \\
\hline 4 & NOTCH4 & 1f & 3 & Thyroid hormone signaling \\
\hline 5 & VCL & 1f & 10 & Shigellosis \\
\hline 6 & PSAP & 1f & 3 & Lysosome \\
\hline 7 & STX2 & 1f & 2 & Synaptic vesicle cycle \\
\hline 8 & GGA2 & 1f & 4 & Lysosome \\
\hline 9 & STK11 & 1f & 7 & Adipocytokine signaling \\
\hline 10 & CSF3R & 1f & 5 & Pathways in cancer \\
\hline 11 & LMNA & 1f & 11 & $\begin{array}{l}\text { Arrhythmogenic right ventricular cardiomyopathy, } \\
\text { Dilated cardiomyopathy, Hypertrophic } \\
\text { cardiomyopathy }\end{array}$ \\
\hline 12 & CTNNA2 & 1f & 3 & Arrhythmogenic right ventricular cardiomyopathy \\
\hline 13 & HLA-C & 1f & 1 & Endocytosis \\
\hline 14 & RAB11FIP4 & 1f & 0 & Endocytosis \\
\hline 15 & GRIN2A & $2 \mathrm{a}$ & 52 & Calcium signaling \\
\hline 16 & RBX1 & $2 \mathrm{a}$ & 0 & Viral Myocarditis \\
\hline 17 & KCNJ5 & $2 \mathrm{a}$ & 0 & Estrogen signaling \\
\hline 18 & EPHA4 & $2 b$ & 18 & Hub Genes \\
\hline 19 & CACNG4 & $2 b$ & 0 & $\begin{array}{l}\text { Arrhythmogenic right ventricular cardiomyopathy, } \\
\text { Dilated cardiomyopathy, Hypertrophic } \\
\text { cardiomyopathy }\end{array}$ \\
\hline 20 & PLA2G5 & $2 b$ & 7 & Pancreatic secretion \\
\hline 21 & ATP2B4 & $2 b$ & 1 & Calcium signaling, pancreatic secretion \\
\hline 22 & P2RY14 & $2 b$ & 0 & Neuroactive ligand receptor interaction \\
\hline 23 & P2RY13 & $2 b$ & 0 & Neuroactive ligand receptor interaction \\
\hline 24 & PTGER4 & $2 b$ & 11 & Neuroactive ligand receptor interaction \\
\hline 25 & ARAP3 & $2 b$ & 0 & Endocytosis \\
\hline 26 & FGF1 & $2 b$ & 22 & Pathways in cancer \\
\hline 27 & RPS6KA2 & $2 b$ & 0 & Neurotrophin signaling \\
\hline 28 & RAPGEF1 & $2 b$ & 0 & Neurotrophin signaling \\
\hline 29 & GABBR2 & $2 b$ & 1 & Estrogen signaling \\
\hline 30 & PRF1 & $2 b$ & 1 & Viral myocarditis \\
\hline 31 & ITGA8 & $2 b$ & 0 & $\begin{array}{l}\text { Arrhythmogenic right ventricular cardiomyopathy, } \\
\text { Dilated cardiomyopathy, Hypertrophic } \\
\text { cardiomyopathy }\end{array}$ \\
\hline 32 & $\mathrm{AP} 2 \mathrm{~A} 2$ & $2 b$ & 0 & Endocytosis, Synaptic vesicle cycle \\
\hline 33 & ITPR2 & $2 b$ & 2 & $\begin{array}{l}\text { Calcium signaling, Estrogen signaling, pancreatic } \\
\text { secretion }\end{array}$ \\
\hline 34 & MED13L & $2 b$ & 0 & Thyroid hormone signaling \\
\hline 35 & COL4A1 & $2 b$ & 0 & Pathways in cancer \\
\hline 36 & KCNJ6 & $2 b$ & 3 & Estrogen signaling \\
\hline 37 & ATP2A3 & $2 b$ & 0 & Calcium signaling, Pancreatic secretion \\
\hline 38 & ASAP2 & $3 a$ & 1 & Endocytosis \\
\hline 39 & FYN & $3 a$ & 70 & Viral myocarditis \\
\hline 40 & NTRK2 & $3 \mathrm{a}$ & 124 & Neurotrophin signaling \\
\hline 41 & PAK1 & $3 a$ & 7 & $\begin{array}{l}\text { Epithelial cell signaling in Helicobacter pylori } \\
\text { infection }\end{array}$ \\
\hline 42 & COL4A2 & $3 a$ & 0 & Small cell lung cancer, Pathways in cancer \\
\hline 43 & BMP4 & $3 \mathrm{a}$ & 5 & Thyroid hormone signaling \\
\hline 44 & GABRB3 & $3 \mathrm{a}$ & 0 & Neuroactive ligand receptor interaction \\
\hline 45 & CEBPB & $3 a$ & 12 & Tuberculosis \\
\hline 46 & EPHA1 & 5 & 31 & Hub Genes \\
\hline 47 & DPYSL2 & 5 & 47 & Hub Genes \\
\hline
\end{tabular}

lation between lower levels of BDNF and cognitive deficits in AD patients was recently reported by Buchman et al. [75]. Recent research work has suggested
BDNF as an upstream regulator of FYN gene, a Src family kinase, leading to enhanced cascade effect of NMDA mediated excitotoxicity and regulates the 
(a)
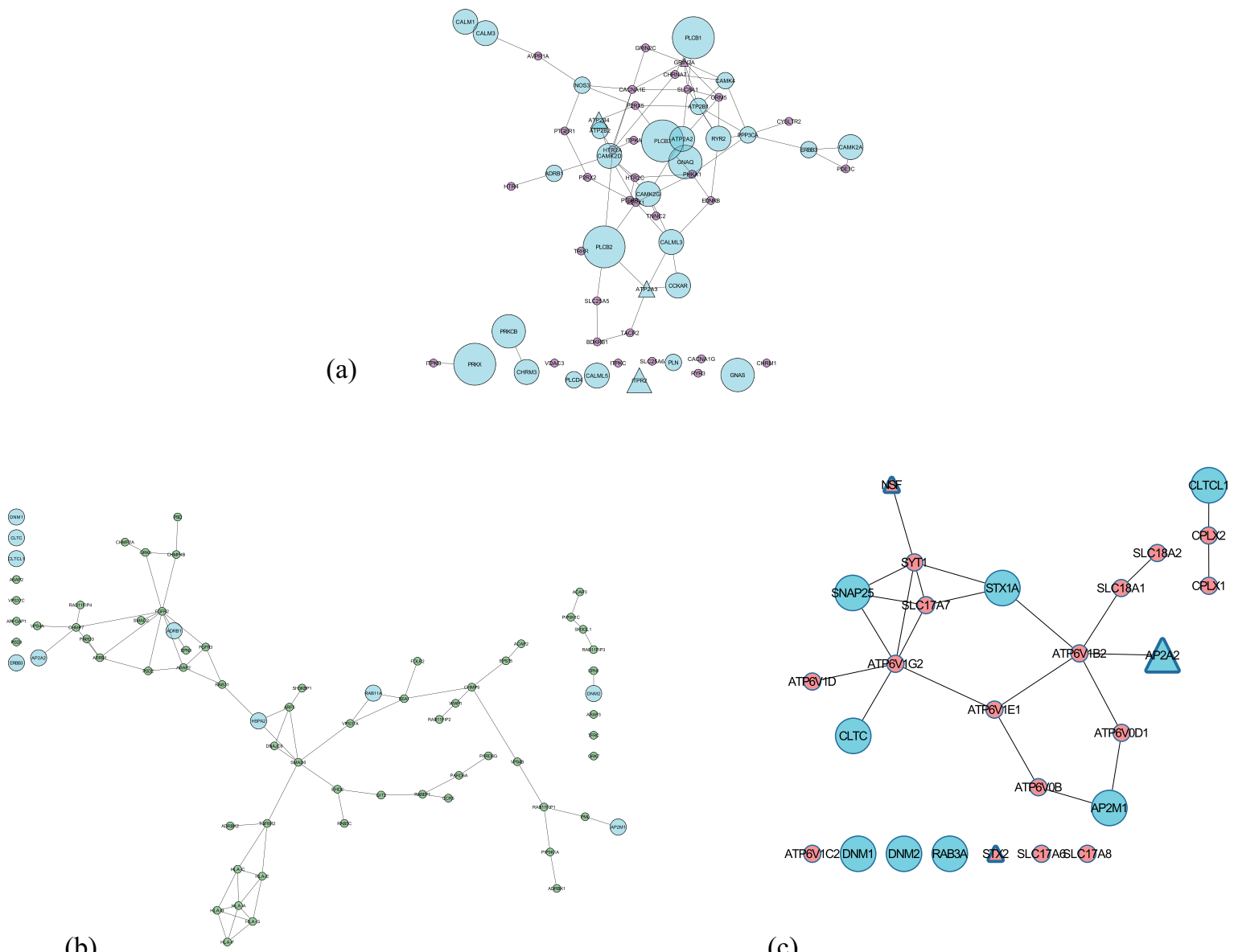

(b)

(c)

Fig. 6. Subnetworks of the three shortlisted potential pathways (extracted from consensus network) involved in neurotransmission. Nodes in Cyan are involved in more than one pathways and the size of the nodes depends on the number of pathways involved. Triangle nodes represent the presence of a SNP. (a) Calcium signaling pathway; (b) Endocytosis pathway; (c) Synaptic vesicle cycle.

activity of hyperphosphorylated tau [76, 77]. In addition, it mediates the synaptic deficits that are induced by $A \beta$ [78]. DPYSL2 mediates synaptic signaling to facilitate neuronal guidance through regulation of calcium channels. Furthermore, FYN phosphorylates DPYSL2 within the brain and its hyperphosphorylation is causally related to $A \beta$ neurotoxicity [79]. Taken together, these findings suggest that synaptic transmission is critical for regulating $A \beta$ production in AD. Further studies, along these lines, may provide insights into the precise molecular mechanism underlying this part of $\mathrm{AD}$ etiology.

\section{Mechanistic interpretation of newly prioritized candidates in neurotransmission}

Neurotransmission is a pivotal brain function that declines with progressing age. However, in the case of
$\mathrm{AD}$, there is a drastic and non-uniform deterioration of synaptic neurotransmission [80]. It is known that soluble oligomeric $A \beta$, rather than insoluble deposits that form plaques (extracellular), are detrimental to synaptic currents through calcium channel modulation, leading to excitotoxic cascades that mediate $\mathrm{AD}$ progression [81] and are related to the formation of neurofibrillary tangles (intracellular) [82]. Emerging research strongly supports the hypothesis of dysregulated calcium homeostasis influencing the presence of neurotoxic $A \beta$ in $A D$ patients [83]. Increased endocytosis activity, enlarged endosomes, has been reported by Cataldo et al. [84] as the earliest intraneuronal neuropathologic feature of $\mathrm{AD}$, subsequently impairing the modulation of NMDA receptor. NMDA excitotoxicity leads to the pathological overload of calcium resulting in synaptic impairment and ultimately neuronal death [85]. 
We observed that three of the "Potential" pathways are significantly involved in neurotransmission: calcium signaling, endocytosis, and synaptic vesicle cycle (see Fig. 6). To assess the modularity of the prioritized candidates in these identified pathways, we extracted the functional relevance of their combination. This confirms our previous findings associated with well-known candidates (see Section Well known prioritized AD candidates). To gain new insights in this context we focused on lesser known prioritized candidates in AD that are involved in these three pathways (less than 5 publications): STX2, HLA-F, HLA-C, RAB11FIP4, ARAP3, AP2A2, ATP2B4, ATP2A3, and ITPR2. Below, we briefly discuss the possibility of these candidates to presumably bear potential as new targets in AD (detailed description is provided in Supplementary File 5).

The presence of $A \beta$ oligomers impairs the process of STX2 binding to SNARE proteins hindering the effective release of neurotransmitter during synaptic vesicle fusion in the presence of increased calcium influx [86, 87]. From several previous studies, one can postulate that HLA-F and HLA-C mediated dysregulated trafficking of amyloid plaques in endocytosis could be correlated to the memory deficits in early AD [88-90]. Several recent evidence point to the fact that faulty $A \beta$ processing can be detected in the membrane trafficking events (linked to RAB11 proteins) of early endosomes, promoting an effective early diagnosis [91, 92]. ARAP3 modulates actin cytoskeleton's remodeling by regulating ARF and RHO family members [93] and a growing body of evidence suggest that axonal transport defects due to its abnormality could be responsible for neurite degeneration and tau toxicity [94-97]. Impairment of APP shuttling by AP2A2 (part of AP-2 complex [98]) from the endocytotic pathway to autophagy degradation leads to intracellular aggregation of $A \beta$ [99]. The next three candidates (ATP2B4, ATP2A3, and ITPR2) participate in neuronal calcium shuttling. A substantial body of evidence indicates ATP2B4, a plasma membrane $\mathrm{Ca}(2+)$ ATPases (PMCAs) is inhibited by A $\beta$ peptides [100], causing cell death [101]. Similarly, ATP2A3's function in handling calcium load and release is perturbed by the mutation in PSEN1 (regulates the intramembrane $A \beta$ processing) [102]. Increased expression of ITPR2 could lead to calcium toxicity in neurons and finally cell death [103, 104].

\section{Conclusion}

The identification of biological mechanisms underlying normal physiology and-when dysregulated-contributing to or even directly causing disease phenotypes is a key objective of current integrative biology. Strategies, both dataand knowledge-driven, for mechanism-identification have shown to deliver valuable insights into disease mechanisms; however, both approaches have their specific drawbacks. Here, we demonstrate a new approach that combines literature-based knowledge and data-driven analysis through gene regulatory networks in a flexible and adaptive way. Thus, allowing us to identify stable and robust patterns of co-expressed genes across several large disparate datasets, in parallel, which enhances the interpretability around "interesting patterns" of co-regulated genes.

We developed an adapted version of BC3Net, called as BC3Net10, that supports a more fine-granular specification of functional context by "injecting" sets of seed genes (derived from literature) into the algorithm. The seed genes were iteratively extended through functional enrichment applied on generated GRNs until convergence. Through several iterations of "selecting and injecting seed genes" and subsequent co-expression analysis, we come up with stable, knowledge-instructed GRNs across several experiments. We show the ability of our approach to identify functional context around subtle signals that would typically be expected for highly individual "modifier" functions not in the core of a dysregulation event, but have the potential to modulate the clinical path of a disease. Hence, making this approach ideally suited for biomarker identification. We show that by the enhanced functional interpretation of the GRNs shed more light on the role of neurotransmission physiology in early dysregulation events presumed to be part of AD etiology. This warrant further investigation of their potential as therapeutic targets.

We would like to point out that there is more potential to the method presented here: in the course of IMI-project AETIONOMY we found limited coverage of signals in knowledge based models coming from the analysis of either gene expression or genetic variation information (GWAS studies). The methodology presented here bears the potential to establish biologically meaningful context around "isolated signals" in knowledge-based models to "embed" previously "non-interpretable" (at functional level) genes 
into a wider (knowledge based) context. Insights drawn from this approach could provide a novel foundation for the formation of new hypotheses. Although microarray data is the obvious starting point, the next logical step would be to extend this work to incorporate orthogonal datatypes such as NGS and single cell data. This could provide a broader view of disease etiology and enable comprehensive in silico investigations. It remains to be shown that the method we introduce here scales up to a really large number of experiments of different sample size.

\section{DISCLOSURE STATEMENT}

The current affiliation of Dr. Philipp Senger is CLS Head of Translational R\&D, BayerCropScience, Alfred-Nobel-Straße 50, 40789 Monheim, Germany. The current affiliation of Mufassra Naz is Institute of Business \& Information Technology, University of Punjab, Lahore. Both contributed to this work during their employment at Fraunhofer SCAI.

Authors' disclosures available online (http://j-alz. com/manuscript-disclosures/17-0011r2).

\section{ACKNOWLEDGMENTS}

We are grateful for the financial support received for this work from the EU/EFPIA Innovative Medicines Initiative Joint Undertaking under AETIONOMY grant agreements $\mathrm{n}^{\circ} 115568$ and $\mathrm{n}^{\circ} 115736$, resources of which are composed of financial contribution from the European Union's Seventh Framework Programme (FP7/2007-2013) and EFPIA companies' in kind contribution.

We thank the anonymous reviewers for their valuable comments.

\section{SUPPLEMENTARY MATERIAL}

The supplementary material is available in the electronic version of this article: http://dx.doi.org/ 10.3233/JAD-170011.

\section{REFERENCES}

[1] Alzheimer's Association (2014) 2014 Alzheimer's disease facts and figures. Alzheimers Dement 10, e47-e92.

[2] Cummings JL, Morstorf T, Zhong K (2014) Alzheimer's disease drug-development pipeline: Few candidates, frequent failures. Alzheimers Res Ther 6, 37.
[3] Andrieu S, Coley N, Lovestone S, Aisen PS, Vellas B (2015) Prevention of sporadic Alzheimer's disease: Lessons learned from clinical trials and future directions. Lancet Neurol 14, 926-944.

[4] Mullane K, Williams M (2013) Alzheimer's therapeutics: Continued clinical failures question the validity of the amyloid hypothesis-but what lies beyond? Biochem Pharmacol 85, 289-305.

[5] Rinne JO, Brooks DJ, Rossor MN, Fox NC, Bullock R, Klunk WE, Mathis CA, Blennow K, Barakos J, Okello AA, Rodriguez Martinez de Liano S, Liu E, Koller M, Gregg KM, Schenk D, Black R, Grundman M (2010) 11C$\mathrm{PiB}$ PET assessment of change in fibrillar amyloid-beta load in patients with Alzheimer's disease treated with bapineuzumab: A phase 2, double-blind, placebo-controlled, ascending-dose study. Lancet Neurol 9, 363-372.

[6] Jones DT, Knopman DS, Gunter JL, Graff-Radford J, Vemuri P, Boeve BF, Petersen RC, Weiner MW, Jack CR, Alzheimer's Disease Neuroimaging Initiative (2016) Cascading network failure across the Alzheimer's disease spectrum. Brain 139, 547-562.

[7] Tseng GC, Ghosh D, Feingold E (2012) Comprehensive literature review and statistical considerations for microarray meta-analysis. Nucleic Acids Res 40, 3785-3799.

[8] Rhodes DR, Yu J, Shanker K, Deshpande N, Varambally R, Ghosh D, Barrette T, Pandey A, Chinnaiyan AM (2004) Large-scale meta-analysis of cancer microarray data identifies common transcriptional profiles of neoplastic transformation and progression. Proc Natl Acad Sci U S A 101, 9309-9314.

[9] Zintzaras E, Ioannidis JPA (2008) Meta-analysis for ranked discovery datasets: Theoretical framework and empirical demonstration for microarrays. Comput Biol Chem 32, 38-46.

[10] Liang D, Han G, Feng X, Sun J, Duan Y, Lei H (2012) Concerted perturbation observed in a hub network in Alzheimer's disease. PLoS One 7, e40498.

[11] Kanehisa M, Goto S, Sato Y, Furumichi M, Tanabe M (2012) KEGG for integration and interpretation of large-scale molecular data sets. Nucleic Acids Res 40, D109-D114.

[12] Liberzon A, Subramanian A, Pinchback R, Thorvaldsdóttir H, Tamayo P, Mesirov JP (2011) Molecular signatures database (MSigDB) 3.0. Bioinformatics 27, 1739-1740.

[13] Langfelder P, Horvath S (2008) WGCNA: An R package for weighted correlation network analysis. BMC Bioinformatics 9, 559 .

[14] de Matos Simoes R, Emmert-Streib F (2012) Bagging statistical network inference from large-scale gene expression data. PLoS One 7, e33624.

[15] Meyer PE, Kontos K, Lafitte F, Bontempi G (2007) Information-theoretic inference of large transcriptional regulatory networks. EURASIP J Bioinform Syst Biol 2007, 79879 .

[16] Margolin AA, Nemenman I, Basso K, Wiggins C, Stolovitzky G, Dalla Favera R, Califano A (2006) ARACNE: An algorithm for the reconstruction of gene regulatory networks in a mammalian cellular context. $B M C$ Bioinformatics 7(Suppl 1), S7.

[17] Huynh-Thu VA, Irrthum A, Wehenkel L, Geurts P (2010) Inferring regulatory networks from expression data using tree-based methods. PLoS One 5, e12776.

[18] Faith JJ, Hayete B, Thaden JT, Mogno I, Wierzbowski J, Cottarel G, Kasif S, Collins JJ, Gardner TS (2007) Large-scale mapping and validation of Escherichia coli 
transcriptional regulation from a compendium of expression profiles. PLOS Biol 5, e8.

[19] de Matos Simoes R, Dalleau S, Williamson KE, EmmertStreib F (2015) Urothelial cancer gene regulatory networks inferred from large-scale RNAseq, Bead and Oligo gene expression data. BMC Syst Biol 9, 21.

[20] de Matos Simoes R, Tripathi S, Emmert-Streib F (2012) Organizational structure and the periphery of the gene regulatory network in B-cell lymphoma. BMC Syst Biol 6, 38 .

[21] Amar D, Safer H, Shamir R (2013) Dissection of regulatory networks that are altered in disease via differential co-expression. PLoS Comput Biol 9, e1002955.

[22] Xu F, Yang J, Chen J, Wu Q, Gong W, Zhang J, Shao W, Mu J, Yang D, Yang Y, Li Z, Xie P (2015) Differential co-expression and regulation analyses reveal different mechanisms underlying major depressive disorder and subsyndromal symptomatic depression. BMC Bioinformatics 16, 112.

[23] Lareau CA, White BC, Oberg AL, McKinney BA (2015) Differential co-expression network centrality and machine learning feature selection for identifying susceptibility hubs in networks with scale-free structure. BioData Min 8,5 .

[24] Djebbari A, Quackenbush J (2008) Seeded Bayesian Networks: Constructing genetic networks from microarray data. BMC Syst Biol 2, 57.

[25] Watson M (2006) CoXpress: Differential co-expression in gene expression data. BMC Bioinformatics 7, 509.

[26] Mukherjee S, Speed TP (2008) Network inference using informative priors. Proc Natl Acad Sci US A 105, 14313-14318.

[27] Ostlund G, Sonnhammer ELL (2014) Avoiding pitfalls in gene (co)expression meta-analysis. Genomics 103, 21-30.

[28] Kim S, Kon M, DeLisi C (2012) Pathway-based classification of cancer subtypes. Biol Direct 7, 21.

[29] Olsen C, Fleming K, Prendergast N, Rubio R, EmmertStreib F, Bontempi G, Haibe-Kains B, Quackenbush J (2014) Inference and validation of predictive gene networks from biomedical literature and gene expression data. Genomics 103, 329-336.

[30] Huang DW, Sherman BT, Lempicki RA (2009) Systematic and integrative analysis of large gene lists using DAVID bioinformatics resources. Nat Protoc 4, 44-57.

[31] Glaab E (2016) Using prior knowledge from cellular pathways and molecular networks for diagnostic specimen classification. Brief Bioinform 17, 440-452.

[32] Bagewadi S, Adhikari S, Dhrangadhariya A, Irin AK, Ebeling C, Namasivayam AA, Page M, Hofmann-Apitius M, Senger P (2015) NeuroTransDB: Highly curated and structured transcriptomic metadata for neurodegenerative diseases. Database (Oxford) 2015, bav099.

[33] Edgar R, Domrachev M, Lash AE (2002) Gene Expression Omnibus: NCBI gene expression and hybridization array data repository. Nucleic Acids Res 30, 207-210.

[34] Parkinson H, Sansone SA, Sarkans U, Rocca-Serra P, Brazma A (2006) ArrayExpress: A public repository for microarray data. Cell Biol Four-Volume Set 4, 95-102.

[35] Zhang B, Gaiteri C, Bodea LG, Wang Z, McElwee J, Podtelezhnikov AA, Zhang C, Xie T, Tran L, Dobrin R, Fluder E, Clurman B, Melquist S, Narayanan M, Suver C, Shah H, Mahajan M, Gillis T, Mysore J, MacDonald ME, Lamb JR, Bennett DA, Molony C, Stone DJ, Gudnason V, Myers AJ, Schadt EE, Neumann H, Zhu J, Emilsson
V (2013) Integrated systems approach identifies genetic nodes and networks in late-onset Alzheimer's disease. Cell 153, 707-720.

[36] CRAN Repository, http://cran.r-project.org/, Accessed on June 25, 2017.

[37] Bollen S (2014) BioConductor: Microarray versus NextGeneration Sequencing toolsets.

[38] Irizarry RA, Hobbs B, Collin F, Beazer-Barclay YD, Antonellis KJ, Scherf U, Speed TP (2003) Exploration, normalization, and summaries of high density oligonucleotide array probe level data. Biostatistics 4, 249-264.

[39] Ritchie ME, Phipson B, Wu D, Hu Y, Law CW, Shi W, Smyth GK (2015) Limma powers differential expression analyses for RNA-sequencing and microarray studies. Nucleic Acids Res 43, e47.

[40] Kauffmann A, Gentleman R, Huber W (2009) arrayQualityMetrics-a bioconductor package for quality assessment of microarray data. Bioinformatics 25, 415-416.

[41] Smoot ME, Ono K, Ruscheinski J, Wang P-L, Ideker T (2011) Cytoscape 2.8: New features for data integration and network visualization. Bioinformatics 27, 431-432.

[42] SCAIView, http://www.scaiview.com, Accesed on June 25, 2017.

[43] Malhotra A, Younesi E, Bagewadi S, Hofmann-Apitius M (2014) Linking hypothetical knowledge patterns to disease molecular signatures for biomarker discovery in Alzheimer's disease. Genome Med 6, 97.

[44] Younesi E, Toldo L, Müller B, Friedrich CM, Novac N, Scheer A, Hofmann-Apitius M, Fluck J (2012) Mining biomarker information in biomedical literature. BMC Med Inform Decis Mak 12, 148.

[45] Fröhlich H (2011) Network based consensus gene signatures for biomarker discovery in breast cancer. PLoS One 6, e25364.

[46] Albright J, Quizon PM, Lusis AJ, Bennett BJ (2014) Genetic network identifies novel pathways contributing to atherosclerosis susceptibility in the innominate artery. BMC Med Genomics 7, 51.

[47] Udyavar AR, Hoeksema MD, Clark JE, Zou Y, Tang Z, Li Z, Li M, Chen H, Statnikov A, Shyr Y, Liebler DC, Field J, Eisenberg R, Estrada L, Massion PP, Quaranta V (2013) Co-expression network analysis identifies Spleen Tyrosine Kinase (SYK) as a candidate oncogenic driver in a subset of small-cell lung cancer. BMC Syst Biol 7(Suppl 5), S1.

[48] Jiang W, Zhang Y, Meng F, Lian B, Chen X, Yu X, Dai E, Wang S, Liu X, Li X, Wang L, Li X (2013) Identification of active transcription factor and miRNA regulatory pathways in Alzheimer's disease. Bioinformatics 29, 25962602.

[49] Kamburov A, Pentchev K, Galicka H, Wierling C, Lehrach H, Herwig R (2011) ConsensusPathDB: Toward a more complete picture of cell biology. Nucleic Acids Res 39, D712-D717.

[50] Carlson M (2015) org. Hs.eg.db: Genome wide annotation for Human. R Packag. version 3.1.2.

[51] Welter D, MacArthur J, Morales J, Burdett T, Hall P, Junkins H, Klemm A, Flicek P, Manolio T, Hindorff L, Parkinson H (2014) The NHGRI GWAS Catalog, a curated resource of SNP-trait associations. Nucleic Acids Res 42, D1001-D1006.

[52] Beck T, Hastings RK, Gollapudi S, Free RC, Brookes AJ (2014) GWAS Central: A comprehensive resource for the comparison and interrogation of genome-wide association studies. Eur J Hum Genet 22, 949-952. 
[53] Li MJ, Wang P, Liu X, Lim EL, Wang Z, Yeager M, Wong MP, Sham PC, Chanock SJ, Wang J (2012) GWASdb: A database for human genetic variants identified by genomewide association studies. Nucleic Acids Res 40, D1047D1054.

[54] Ward LD, Kellis M (2012) HaploReg: A resource for exploring chromatin states, conservation, and regulatory motif alterations within sets of genetically linked variants. Nucleic Acids Res 40, D930-D934.

[55] Sherry ST, Ward MH, Kholodov M, Baker J, Phan L, Smigielski EM, Sirotkin K (2001) dbSNP: The NCBI database of genetic variation. Nucleic Acids Res 29, 308-311.

[56] Pazin MJ (2015) Using the ENCODE resource for functional annotation of genetic variants. Cold Spring Harb Protoc 2015, 522-536.

[57] Chadwick LH (2012) The NIH roadmap epigenomics program data resource. Epigenomics 4, 317-324.

[58] Gibson G (2015) Human genetics. GTEx detects genetic effects. Science 348, 640-641.

[59] McLaren W, Pritchard B, Rios D, Chen Y, Flicek P, Cunningham F (2010) Deriving the consequences of genomic variants with the Ensembl API and SNP Effect Predictor. Bioinformatics 26, 2069-2070.

[60] Boyle AP, Hong EL, Hariharan M, Cheng Y, Schaub M a., Kasowski M, Karczewski KJ, Park J, Hitz BC, Weng S, Cherry JM, Snyder M (2012) Annotation of functional variation in personal genomes using RegulomeDB. Genome Res 22, 1790-1797.

[61] ENSEMBL's Variant Effect Predictor, http://www. ensembl.org/info/docs/tools/vep/index.html, Accessed on June 25, 2017.

[62] ENSEMBL Variantion Database, http://www.ensembl. org/info/genome/variation/index.html, Accessed on June 25, 2017

[63] Xie D, Boyle AP, Wu L, Zhai J, Kawli T, Snyder M (2013) Dynamic trans-acting factor colocalization in human cells. Cell 155, 713-724.

[64] Boyle AP, Song L, Lee B-K, London D, Keefe D, Birney E, Iyer VR, Crawford GE, Furey TS (2011) High-resolution genome-wide in vivo footprinting of diverse transcription factors in human cells. Genome Res 21, 456-464.

[65] Jolma A, Yan J, Whitington T, Toivonen J, Nitta KR, Rastas P, Morgunova E, Enge M, Taipale M, Wei G, Palin K, Vaquerizas JM, Vincentelli R, Luscombe NM, Hughes TR, Lemaire P, Ukkonen E, Kivioja T, Taipale J (2013) DNAbinding specificities of human transcription factors. Cell 152, 327-339.

[66] Kuleshov V, Xie D, Chen R, Pushkarev D, Ma Z, Blauwkamp T, Kertesz M, Snyder M (2014) Wholegenome haplotyping using long reads and statistical methods. Nat Biotechnol 32, 261-266.

[67] He X, Zhang J (2006) Why do hubs tend to be essential in protein networks? PLoS Genet 2 , e88.

[68] Lambert JC, Ibrahim-Verbaas CA, Harold D, Naj AC, Sims R, Bellenguez C, DeStafano AL, Bis JC, Beecham GW, Grenier-Boley B, Russo G, Thorton-Wells TA, Jones N, Smith AV, Chouraki V, Thomas C, Ikram MA, Zelenika D, Vardarajan BN, Kamatani Y, Lin CF, Gerrish A, Schmidt H, Kunkle B, Dunstan ML, Ruiz A, Bihoreau MT, Choi SH, Reitz C, Pasquier F, Cruchaga C, Craig D, Amin N, Berr C, Lopez OL, De Jager PL, Deramecourt V, Johnston JA, Evans D, Lovestone S, Letenneur L, Morón FJ, Rubinsztein DC, Eiriksdottir G, Sleegers K, Goate AM, Fiévet N,
Huentelman MW, Gill M, Brown K, Kamboh MI, Keller L, Barberger-Gateau P, McGuiness B, Larson EB, Green R, Myers AJ, Dufouil C, Todd S, Wallon D, Love S, Rogaeva E, Gallacher J, St George-Hyslop P, Clarimon J, Lleo A, Bayer A, Tsuang DW, Yu L, Tsolaki M, Bossù P, Spalletta G, Proitsi P, Collinge J, Sorbi S, Sanchez-Garcia F, Fox NC, Hardy J, Deniz Naranjo MC, Bosco P, Clarke R, Brayne C, Galimberti D, Mancuso M, Matthews F, European Alzheimer's Disease Initiative (EADI), Genetic, Environmental Risk in Alzheimer's Disease, Alzheimer's Disease Genetic Consortium, Cohorts for Heart, Aging Research in Genomic Epidemiology, Moebus S, Mecocci P, Del Zompo M, Maier W, Hampel H, Pilotto A, Bullido M, Panza F, Caffarra P, Nacmias B, Gilbert JR, Mayhaus M, Lannefelt L, Hakonarson H, Pichler S, Carrasquillo MM, Ingelsson M, Beekly D, Alvarez V, Zou F, Valladares O, Younkin SG, Coto E, Hamilton-Nelson KL, Gu W, Razquin C, Pastor P, Mateo I, Owen MJ, Faber KM, Jonsson PV, Combarros O, O'Donovan MC, Cantwell LB, Soininen H, Blacker D, Mead S, Mosley TH, Bennett DA, Harris TB, Fratiglioni L, Holmes C, de Bruijn RF, Passmore P, Montine TJ, Bettens K, Rotter JI, Brice A, Morgan K, Foroud TM, Kukull WA, Hannequin D, Powell JF, Nalls MA, Ritchie K, Lunetta KL, Kauwe JS, Boerwinkle E, Riemenschneider M, Boada M, Hiltuenen M, Martin ER, Schmidt R, Rujescu D, Wang LS, Dartigues JF, Mayeux R, Tzourio C, Hofman A, Nöthen MM, Graff C, Psaty BM, Jones L, Haines JL, Holmans PA, Lathrop M, Pericak-Vance MA, Launer LJ, Farrer LA, van Duijn CM, Van Broeckhoven C, Moskvina V, Seshadri S, Williams J, Schellenberg GD, Amouyel P (2013) Meta-analysis of 74,046 individuals identifies 11 new susceptibility loci for Alzheimer's disease. Nat Genet 45, 1452-1458.

[69] Di Bona D, Plaia A, Vasto S, Cavallone L, Lescai F, Franceschi C, Licastro F, Colonna-Romano G, Lio D, Candore G, Caruso C (2008) Association between the interleukin-1beta polymorphisms and Alzheimer's disease: A systematic review and meta-analysis. Brain Res Rev 59, 155-163.

[70] Bathina S, Das UN (2015) Brain-derived neurotrophic factor and its clinical implications. Arch Med Sci 11, 1164-1178.

[71] Yallapu MM, Jaggi M, Chauhan SC (2012) Curcumin nanoformulations: A future nanomedicine for cancer. Drug Discov Today 17, 71-80.

[72] Ye X, Tai W, Zhang D (2012) The early events of Alzheimer's disease pathology: From mitochondrial dysfunction to BDNF axonal transport deficits. Neurobiol Aging 33, 1122.e1-10.

[73] Nizzari M, Thellung S, Corsaro A, Villa V, Pagano A, Porcile C, Russo C, Florio T (2012) Neurodegeneration in Alzheimer disease: Role of amyloid precursor protein and presenilin 1 intracellular signaling. J Toxicol 2012, 187297.

[74] Marambaud P, Dreses-Werringloer U, Vingtdeux V (2009) Calcium signaling in neurodegeneration. Mol Neurodegener 4, 20.

[75] Buchman AS, Yu L, Boyle PA, Schneider JA, De Jager PL, Bennett DA (2016) Higher brain BDNF gene expression is associated with slower cognitive decline in older adults. Neurology 86, 735-741.

[76] Yang K, Belrose J, Trepanier CH, Lei G, Jackson MF, MacDonald JF (2011) Fyn, a potential target for Alzheimer's disease. J Alzheimers Dis 27, 243-252. 
[77] Yallapu MM, Jaggi M, Chauhan SC (2012) Curcumin nanoformulations: A future nanomedicine for cancer. Drug Discov Today 17, 71-80.

[78] Wang H, Ren CH, Gunawardana CG, Schmitt-Ulms G (2013) Overcoming barriers and thresholds - signaling of oligomeric A $\beta$ through the prion protein to Fyn. Mol Neurodegener $\mathbf{8}, 24$.

[79] Isono T, Yamashita N, Obara M, Araki T, Nakamura F, Kamiya Y, Alkam T, Nitta A, Nabeshima T, Mikoshiba K, Ohshima T, Goshima Y (2013) Amyloid- $\beta_{25-35}$ induces impairment of cognitive function and long-term potentiation through phosphorylation of collapsin response mediator protein 2. Neurosci Res 77, 180-185.

[80] Wang X, Michaelis ML, Michaelis EK (2010) Functional genomics of brain aging and Alzheimer's disease: Focus on selective neuronal vulnerability. Curr Genomics 11, 618633.

[81] Hermann D, Mezler M, Müller MK, Wicke K, Gross G, Draguhn A, Bruehl C, Nimmrich V (2013) Synthetic $A \beta$ oligomers ( $A \beta(1-42)$ globulomer) modulate presynaptic calcium currents: Prevention of $A \beta$-induced synaptic deficits by calcium channel blockers. Eur J Pharmacol 702, 44-55.

[82] Roberson ED, Scearce-Levie K, Palop JJ, Yan F, Cheng IH, Wu T, Gerstein H, Yu G-Q, Mucke L (2007) Reducing endogenous tau ameliorates amyloid beta-induced deficits in an Alzheimer's disease mouse model. Science 316, 750-754.

[83] Bezprozvanny I, Mattson MP (2008) Neuronal calcium mishandling and the pathogenesis of Alzheimer's disease. Trends Neurosci 31, 454-463.

[84] Cataldo AM, Peterhoff CM, Troncoso JC, Gomez-Isla T, Hyman BT, Nixon RA (2000) Endocytic pathway abnormalities precede amyloid beta deposition in sporadic Alzheimer's disease and Down syndrome: Differential effects of APOE genotype and presenilin mutations. Am J Pathol 157, 277-286.

[85] Danysz W, Parsons CG (2012) Alzheimer's disease, $\beta$-amyloid, glutamate, NMDA receptors and memantinesearching for the connections. Br J Pharmacol 167, 324-352.

[86] Ghiani CA, Starcevic M, Rodriguez-Fernandez IA, Nazarian R, Cheli VT, Chan LN, Malvar JS, de Vellis J, Sabatti C, Dell'Angelica EC (2010) The dysbindin-containing complex (BLOC-1) in brain: Developmental regulation, interaction with SNARE proteins and role in neurite outgrowth. Mol Psychiatry 15 115, 204-215.

[87] Band AM, Kuismanen E (2005) Localization of plasma membrane t-SNAREs syntaxin 2 and 3 in intracellular compartments. BMC Cell Biol 6, 26.

[88] de Groot CJ, Hulshof S, Hoozemans JJ, Veerhuis R (2001) Establishment of microglial cell cultures derived from postmortem human adult brain tissue: Immunophenotypical and functional characterization. Microsc Res Tech 54, 34-39.

[89] McGeer PL, McGeer EG (2013) The amyloid cascadeinflammatory hypothesis of Alzheimer disease: Implications for therapy. Acta Neuropathol 126, 479-497.

[90] Nelson PA, Sage JR, Wood SC, Davenport CM, Anagnostaras SG, Boulanger LM (2013) MHC class I immune proteins are critical for hippocampus-dependent memory and gate NMDAR-dependent hippocampal long-term depression. Learn Mem 20, 505-517.

[91] Thompson AJ, Williamson R, Schofield E, Stephenson J, Hanger D, Anderton B (2009) Quantitation of glycogen synthase kinase-3 sensitive proteins in neuronal membrane rafts. Proteomics 9, 3022-3035.

[92] Chia PZC, Toh WH, Sharples R, Gasnereau I, Hill AF, Gleeson PA (2013) Intracellular itinerary of internalised $\beta$ secretase, BACE1, and its potential impact on $\beta$-amyloid peptide biogenesis. Traffic 14, 997-1013.

[93] Porat-Shliom N, Milberg O, Masedunskas A, Weigert R (2013) Multiple roles for the actin cytoskeleton during regulated exocytosis. Cell Mol Life Sci 70, 2099-2121.

[94] De Vos KJ, Grierson AJ, Ackerley S, Miller CCJ (2008) Role of axonal transport in neurodegenerative diseases. Annu Rev Neurosci 31, 151-173.

[95] Yallapu MM, Jaggi M, Chauhan SC (2012) Curcumin nanoformulations: A future nanomedicine for cancer. Drug Discov Today 17, 71-80.

[96] Fulga TA, Elson-Schwab I, Khurana V, Steinhilb ML, Spires TL, Hyman BT, Feany MB (2007) Abnormal bundling and accumulation of F-actin mediates tauinduced neuronal degeneration in vivo. Nat Cell Biol 9, 139-148.

[97] Halstead JM, Lionnet T, Wilbertz JH, Wippich F, Ephrussi A, Singer RH, Chao JA (2015) Translation. An RNA biosensor for imaging the first round of translation from single cells to living animals. Science 347, 1367-1671.

[98] UniProt Database, http://www.uniprot.org/, Accessed on June 25, 2017.

[99] Tian Y, Chang JC, Fan EY, Flajolet M, Greengard P (2013) Adaptor complex AP2/PICALM, through interaction with LC3, targets Alzheimer's APP-CTF for terminal degradation via autophagy. Proc Natl Acad Sci U S A 110, 17071-17076.

[100] Berrocal M, Sepulveda MR, Vazquez-Hernandez M, Mata AM (2012) Calmodulin antagonizes amyloid- $\beta$ peptidesmediated inhibition of brain plasma membrane $\mathrm{Ca}(2+)$ ATPase. Biochim Biophys Acta 1822, 961-969.

[101] Mata AM, Berrocal M, Sepúlveda MR (2011) Impairment of the activity of the plasma membrane $\mathrm{Ca} 2+$ ATPase in Alzheimer's disease. Biochem Soc Trans 39, 819-822.

[102] Green KN, LaFerla FM (2008) Linking calcium to Abeta and Alzheimer's disease. Neuron 59, 190-194.

[103] Kaja S, Duncan RS, Longoria S, Hilgenberg JD, Payne AJ, Desai NM, Parikh RA, Burroughs SL, Gregg EV, Goad DL, Koulen P (2011) Novel mechanism of increased $\mathrm{Ca} 2+$ release following oxidative stress in neuronal cells involves type 2 inositol-1,4,5-trisphosphate receptors. Neuroscience 175, 281-291.

[104] Jiang P, Chen C, Liu X-B, Selvaraj V, Liu W, Feldman DH, Liu Y, Pleasure DE, Li RA, Deng W (2013) Generation and characterization of spiking and nonspiking oligodendroglial progenitor cells from embryonic stem cells. Stem Cells 31, 2620-2631. 